\title{
Quenched nematic criticality and two superconducting domes in an iron-based superconductor
}

\author{
P. Reiss, ${ }^{1, *}$ D. Graf, ${ }^{2}$ A. A. Haghighirad,${ }^{1,3}$ W. Knafo, ${ }^{4}$ L. Drigo,,${ }^{4,5}$ M. Bristow, ${ }^{1}$ A. J. Schofield,${ }^{6}$ and A. I. Coldea ${ }^{1, \dagger}$ \\ ${ }^{1}$ Clarendon Laboratory, Department of Physics, University of Oxford, Parks Road, Oxford OX1 3PU, UK \\ ${ }^{2}$ National High Magnetic Field Laboratory and Department of Physics, \\ Florida State University, Tallahassee, Florida 32306, USA \\ ${ }^{3}$ Institut für Festkörperphysik, Karlsruhe Institute of Technology, 76021 Karlsruhe, Germany \\ ${ }^{4}$ Laboratoire National des Champs Magnétiques Intenses (LNCMI-EMFL), \\ UPR 3228, CNRS-UJF-UPS-INSA, 143 Avenue de Rangueil, 31400 Toulouse, France \\ ${ }^{5}$ Géosciences Environnement Toulouse (CNRS), 31400 Toulouse, France \\ ${ }^{6}$ School of Physics and Astronomy, University of Birmingham, Edgbaston, Birmingham B15 2TT, UK
}

\begin{abstract}
The nematic electronic state and its associated critical fluctuations have emerged as a potential candidate for the superconducting pairing in various unconventional superconductors. However, in most materials their coexistence with magnetically-ordered phases poses a significant challenge in determining their importance. Here, by combining chemical and hydrostatic physical pressure in $\mathrm{FeSe}_{0.89} \mathrm{~S}_{0.11}$, we access a nematic quantum phase transition isolated from any other competing magnetic phases. From quantum oscillations in high magnetic fields, we trace the evolution of the Fermi surface and electronic correlations as a function of applied pressure and detect a Lifshitz transition that separates two distinct superconducting regions. One emerges from the nematic phase with a small Fermi surface and strong electronic correlations, while the other has a large Fermi surface and weak correlations that promotes nesting and stabilization of a magnetically-ordered phase at high pressures. The absence of mass divergence at the nematic quantum phase transition suggests that the nematic fluctuations could be quenched by the strong coupling to the lattice or local strain effects. A direct consequence is the weakening of superconductivity at the nematic quantum phase transition in the absence of magnetically driven fluctuations.
\end{abstract}

Quantum materials brought in the vicinity of a quantum critical point at absolute zero temperature have generated significant interest in condensed matter physics as they can trigger the emergence of novel electronic states [1]. In these extreme regimes, achieved by tuning external parameters, the nature of quasiparticles is significantly altered due to the interactions with critical fluctuations and non-Fermi liquid behaviour is often observed [2]. A particulary interesting instability of a metallic system is the electronic nematic state. In such a state, the electron motion spontaneously breaks the rotational symmetry of the crystal in the presence of strong electronic interactions and its Fermi surface undergoes a spontaneous distortion [3]. The observation of electronic nematic order in different families of high-temperature superconductors implies that the same interactions may be involved in stabilizing both the nematic and superconducting states [4-6]. However, the presence of other competing electronic phases, such as spin or charge-density waves neighbouring superconductivity can obscure the relevance of the nematic fluctuations in superconducting pairing.

FeSe is an example of a nematic superconductor in which applied pressure leads to a four-fold increase in its bulk superconductivity (from $9 \mathrm{~K}$ towards a high-critical temperature, $\left.T_{c} \sim 40 \mathrm{~K}\right)[7,8]$. In the normal state, the nematic phase transition of FeSe is suppressed with increasing pressure, but the quantum phase transition is masked by an emerging magnetic ordering stabilized under high pressures [9-16]. As the nematic and magnetic phases of FeSe are intertwined, it is difficult to establish the roles played by nematic or spin fluctuations for stabilizing the high- $T_{c}$ state. Similarly, isoelectronic sulfur substitution in $\mathrm{FeSe}_{1-x} \mathrm{~S}_{x}$, equivalent to internal positive pressure, suppresses the nematic order, but does not stabilize a magnetically ordered state [17-21]. Consequently, by combining chemical pressure and hydrostatic pressure, a nematic quantum critical point can be unmasked, as the magnetic order is shifted to higher pressures with increasing sulfur concentration [21-24]. This opens a unique path for studying the nature of nematic criticality using hydrostatic pressure as a clean tuning parameter and to probe the role of nematic fluctuations in stabilizing superconductivity.

As superconductivity depends not only on the origin of the attractive pairing interaction but also on the details of the Fermi surface [25], understanding the changes in the electronic structure as a function of different tuning parameters is paramount. Furthermore, the size of the Fermi energy can trigger different electronic instabilities: a nematic order for small energies, superconductivity in an intermediate regime or a magnetically-ordered state for large energies [26]. To test these different regimes, quantum oscillations are a powerful technique to access the evolution of the electronic structure and correlations under extreme conditions.

In this Article we explore the electronic behaviour across the pressure-temperature phase diagram of $\mathrm{FeSe}_{0.89} \mathrm{~S}_{0.11}$, in the absence of long-range magnetic order. Using external pressure, we finely tune the system across its nematic phase transition to understand the nature of the critical regime and to establish the role played by the electronic correlations, electronic structure and scattering in stabilizing superconductivity. We identify an extended region with a $\sim T^{3 / 2}$ resistivity which evolves into a Fermi liquid behaviour at low temperatures in the vicinity of the nematic phase transition. We find that the cyclotron effective masses from quantum oscillations 
are significantly suppressed across the nematic phase transition, as is superconductivity. This behaviour could be a signature of quenched critical fluctuations due to a nematoelastic coupling to the lattice [27], and/or local strain effects.

Temperature dependence of resistivity with applied pressure. Figure 1A shows the evolution of the temperature dependence of the resistivity as a function of applied pressure below $100 \mathrm{~K}$ (the full range up to $300 \mathrm{~K}$ is shown in Fig. S1 in the Supplemental Information (SI)). With increasing pressure, the nematic transition temperature $T_{s}$ is quickly suppressed from $60 \mathrm{~K}$ at ambient pressure until it becomes unobservable around $p_{c}$ (Fig. S1 in the $\mathrm{SI}$ ) and no hysteresis between warming and cooling at constant pressure is detected (see Fig. S9 in the SI). By extrapolating the pressure evolution of $T_{s}$ to zero, we can locate the position of the nematic quantum phase transition at $p_{c} \sim 5.8(5) \mathrm{kbar}$ in Fig. 1E. Within the nematic phase, there is only a weak pressure dependence of the superconducting transition temperature $T_{c}$ (defined as the zero resistivity temperature) similar to previous findings using chemical pressure $[19,20]$. Outside the nematic phase, superconductivity is suppressed rather than enhanced in the proximity of the nematic quantum phase transition and reaches a minimum $T_{c} \sim 6.4 \mathrm{~K}$ (at $\sim 7 \mathrm{kbar}$ ) close to $p_{c}$. However, superconductivity is enhanced significantly towards $22 \mathrm{~K}$ at $20 \mathrm{kbar}$, and $T_{c}$ was reported to reach $30 \mathrm{~K}$ at higher pressures at around $30 \mathrm{kbar}$ [22]. The superconducting transition at high pressures broadens significantly compared with the low-pressure region (by a factor $\sim 10$ ), suggesting that the superconducting phase becomes rather inhomogeneous at high pressures or that it coexists with another electronic phase. In contrast to FeSe where nematicity, magnetism and superconductivity may coexist under pressure [9, 13, 23], magnetic order in our composition is expected to be stabilized only at high pressures exceeding $40 \mathrm{kbar}$ [22].

To elucidate the nature of quasi-particle scattering in the proximity of the nematic quantum phase transition, we investigate the resistivity exponent $n$, defined as $\rho(T)=\rho_{0}+A T^{n}$, as a function of pressure and temperature. For a non- or weakly interacting Fermi liquid, one expects $n=2$. In contrast, for systems in the proximity of quantum critical points, enhanced order parameter fluctuations can lead to non-Fermi liquid behaviour with an exponent $n<2$ and additional scattering $[5,30,31]$. The precise power law is influenced by the nature of the critical fluctuations (their wave-vector and dynamical exponent), by dimensionality [32], by the amount of disorder [30] and by nearby quantum critical points [33]. The nematic critical fluctuations can be exceedingly effective in destroying quasiparticles and can produce a striking nodal-antinodal dichotomy generating non-Fermi liquid-like behaviour over much of the Fermi surface enhancing resistivity [5, 34]. At low temperatures away form the critical points in a clean system, Fermi-liquid like behaviour is often recovered $[32,35]$. In this regime, the $A$ coefficient, which is a measure of the strength of the electronic correlations as $A \sim\left(m^{*} / m_{e}\right)^{2}$, diverges upon approaching the critical region as fluctuations become critical $\left(m^{*}\right.$ is the quasiparticle
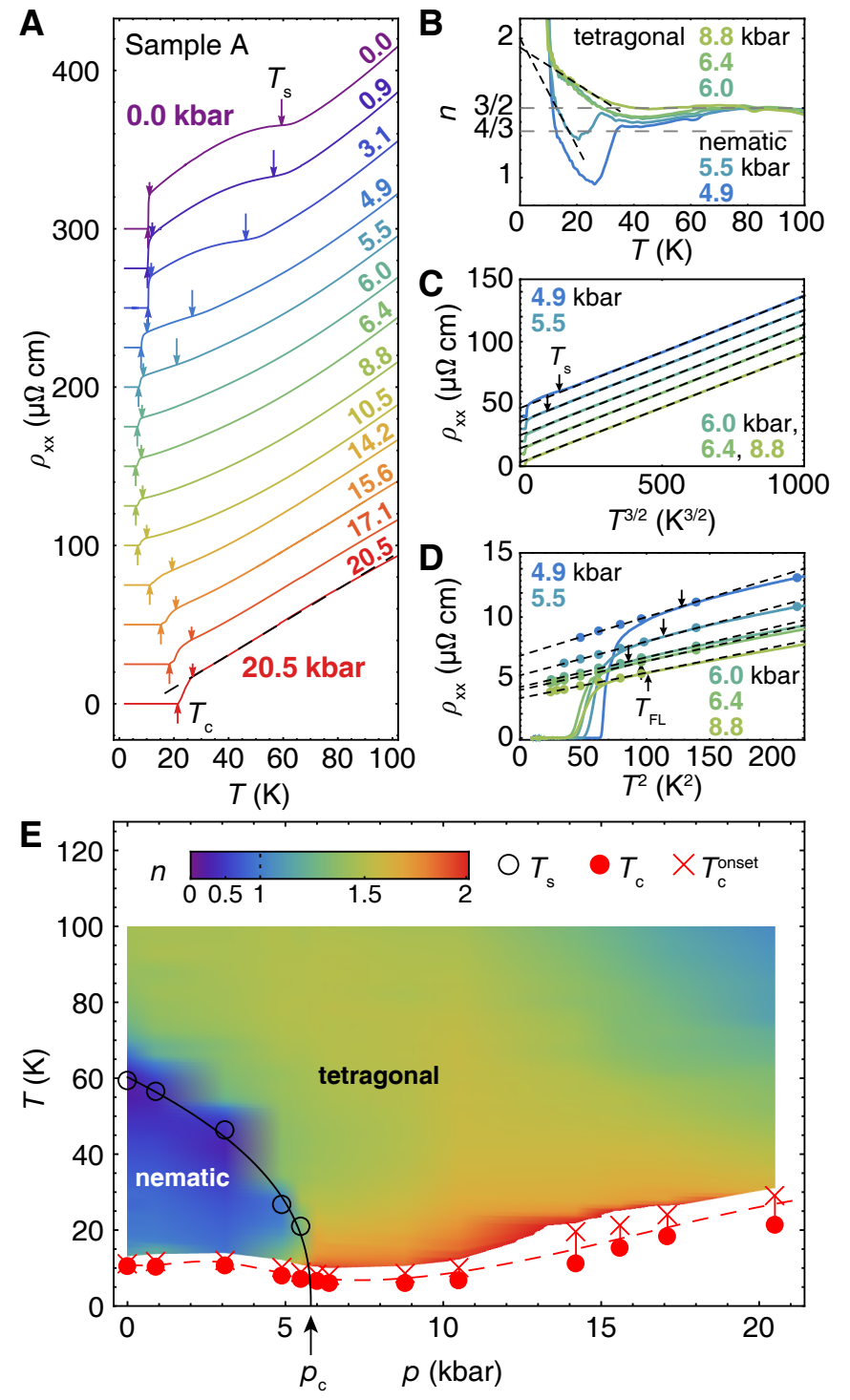

FIG. 1. Transport under pressure in $\mathbf{F e S e}_{0.89} \mathbf{S}_{0.11}$. (A) Temperature dependence of resistivity for different pressures. The transition temperature into the nematic state occurs at $T_{s}$ and into the superconducting phase at $T_{c}$. The onset and offset of superconductivity are indicated by crosses and solid circles, respectively in (E). (B) Local resistivity exponent $n$ for pressures close to $p_{c}$. Dashed lines are linear fits at low temperatures above the onset of superconductivity. (C) High temperature resistivity plotted against $T^{3 / 2}$. Dashed lines are linear fits. Data in panels $\mathbf{A}$ and $\mathbf{C}$ are shifted vertically for clarity. (D) Low-temperature resistivity (solid lines) and normal state resistivity extrapolated from symmetrized magnetic field measurements (points) plotted against $T^{2}$ (Fig.S2). Dashed lines are fits to Fermi liquid behaviour, $\rho=\rho_{0}+A T^{2}$, the slope gives the $A$ coefficient and $\rho_{0}$ is the zero-temperature residual resistivity. Fit residuals are shown in Fig. S3. (E) Pressure-temperature phase diagram. The color map represents the local resistivity exponent $n\left(\rho \sim T^{n}\right)$, see color scale in inset. Symbols indicate the transition temperatures defined in panel (A). The dashed line is a guide to the eye. The solid line is a fit of $T_{s} \sim\left(p-p_{c}\right)^{\epsilon}$ giving $\epsilon \sim 0.4(1)$ and $p_{c}=5.8(5) \mathrm{kbar}$. To establish the value of the critical exponent, detailed pressure points close to $p_{c}$ are required, as detailed in Ref. [28]. 

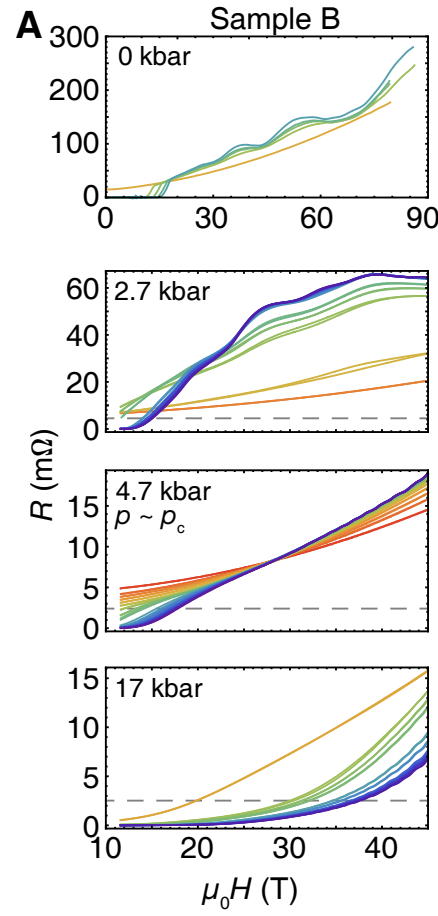
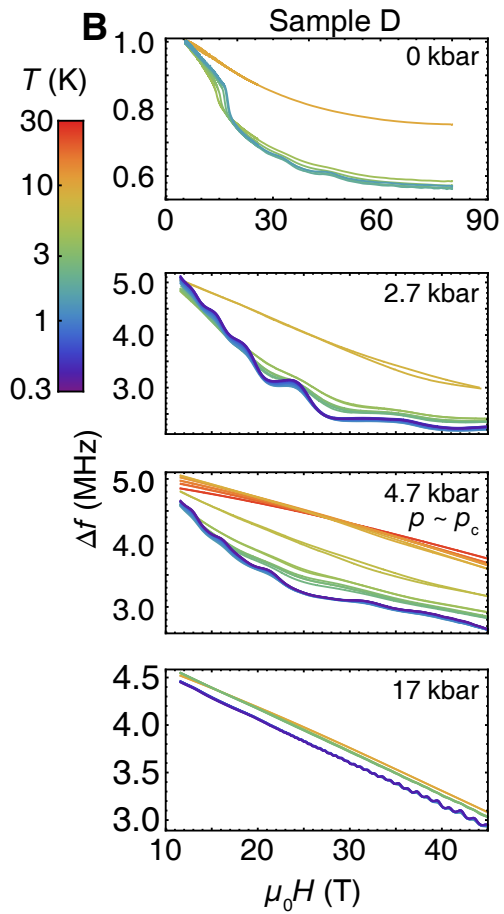
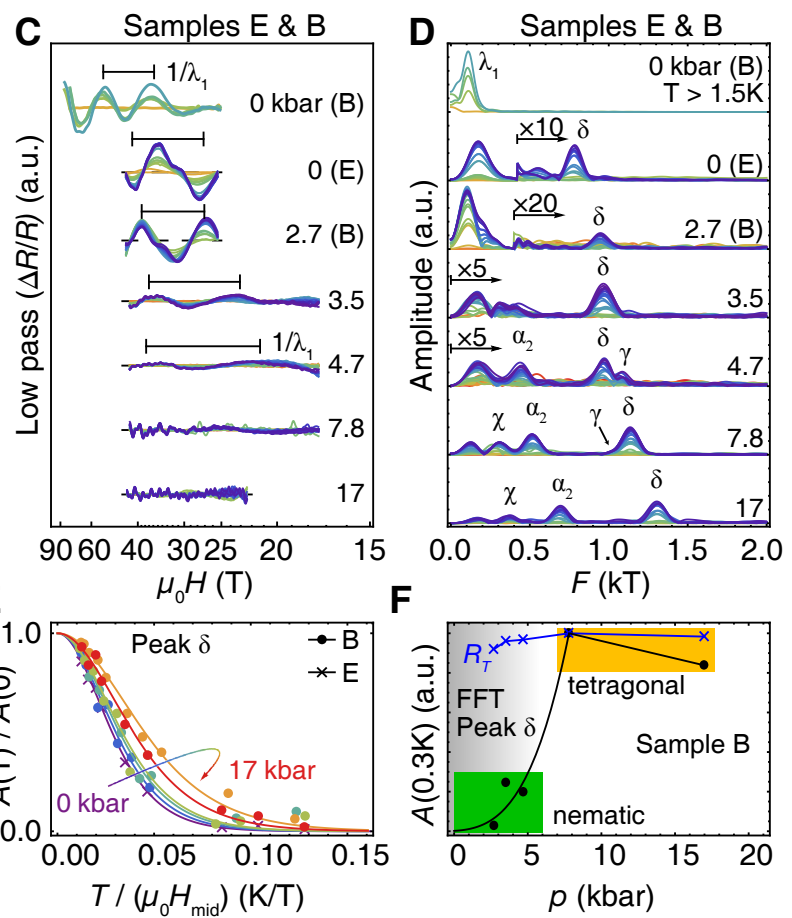

FIG. 2. Evolution of quantum oscillations with pressure in $\mathbf{F e S e}_{0.89} \mathbf{S}_{0.11}$. $\quad$ (A) Magnetotransport and (B) TDO resonant frequency variation, $\Delta f(\mathrm{MHz})$, for several different samples as a function of applied pressures (see also Figs. S4-S6). Horizontal dashed lines in $\mathbf{A}$ indicate the sample resistance in zero field at the onset of superconductivity. (C) The oscillatory component of the magnetoresistance (after subtracting of a smooth background with a low-pass filter applied) shows the evolution of the dominant low-frequency oscillation. Dashed lines here indicate the base line. The horizontal bars indicate the period of the dominant low frequency $\left(\lambda_{1}\right)$. (D) Fourier transform of the oscillatory part of the magnetotransport data in (A) after the background subtraction. The distinct peaks identified correspond to extremal cross-sections of the Fermi surface. (E) The temperature dependence of the amplitude gives the cyclotron effective band mass of the $\delta$ orbit for different pressures. Lines are fits to the Lifshitz-Kosevich mass damping term [29]. (F) Low-temperature amplitude variation with pressure of the $\delta$ orbit (solid symbols) and the expected amplitude variation due to the mass damping term $R_{T}$ (cross symbols). Solid lines are guides to the eye. Data in panels $\mathbf{C}$ and $\mathbf{D}$ are shifted vertically for clarity.

effective mass) [36, 37].

Figure $1 \mathrm{E}$ shows the temperature and pressure dependence of $n$ as a color map. Near the nematic end point at $p_{c}$, the resistivity does not follow a linear $T$ dependence, as found near magnetic critical points [36] nor does it display the expected quantum critical fan with a constant exponent [32]. Instead, this region is best represented by a temperature-dependent resistivity exponent $n$, shown in Fig.1B, that evolves from a value around $n=3 / 2$ at high temperatures towards the $n=2$ value in the low temperature regime. In fact, $n$ shows a marked upturn below $30 \mathrm{~K}$ for pressures close to $p_{c}$ that can be linearly extrapolate towards $n=2$, as shown by dashed lines in Fig. 1B. To further demonstrate this result, we can use magnetic fields to suppress superconductivity. At the lowest temperatures below $15 \mathrm{~K}$, we find a Fermi-liquid behaviour for all pressures across $p_{c}$, as shown in Fig. 1D (see also Figs. S1 and S3 in the SI). Furthermore, the corresponding $A$ coefficient as well as the zero-temperature resistivity, $\rho_{0}$, are continuously suppressed with increasing pressure across the transition (Figs. 4B and $\mathrm{S} 1$ in the $\mathrm{SI}$ ). This strongly suggests that at $p_{c}$ the nematic fluctuations do not become critical.

The evolution of the Fermi surface with pressure. Next, we use quantum oscillations measurements under pressure to follow the evolution of the Fermi surface and to assess the strength of electronic correlations across the phase diagram of $\mathrm{FeSe}_{0.89} \mathrm{~S}_{0.11}$ under pressure. Figures $2 \mathrm{~A}$ and $\mathrm{B}$ show the field dependence of the resistance and tunnel diode oscillator (TDO) data for pressures up to $17 \mathrm{kbar}$ and up to $45 \mathrm{~T}$, and the ambient pressure measurements up to $80 \mathrm{~T}$ for three different samples. Results on additional samples and pressures are shown in Figs. S4-S8 in the SI. There are different field regimes seen in the raw data: a) the superconducting state with zero resistance at low magnetic fields, b) finite resistance that increases strongly with magnetic field in the crossover vortexliquid region to the normal state at higher fields and c) normal magnetoresistance accompanied by quantum oscillations in high magnetic fields.

At low pressures in the nematic state, the magnetotransport and TDO data is dominated by a low-frequency oscillation (Fig. 2A) that disappears beyond $4.7 \mathrm{kbar}$, in the proximity of $p_{c}$ (Figs. 2A, B and C). At high pressures and in high fields, a high-frequency oscillation is visible in the raw data (see Figs. 2A and B), associated with a large Fermi surface sheet. This rules out a reconstruction of the Fermi surface 
at high pressures, outside the nematic phase, similar to the tetragonal phases of $\mathrm{FeSe}_{1-x} \mathrm{~S}_{x}$ [19], but in contrast to FeSe under pressure inside the magnetic phase [10].

Quantum oscillation spectra of $\mathrm{FeSe}_{0.89} \mathrm{~S}_{0.11}$ reveal several distinct peaks, consistent with a complex multi-band electronic structure, as shown in Fig. 2D. Based on ARPES data and previous quantum oscillation measurements, the ambient pressure Fermi surface is formed of two concentric electronlike and one outer hole-like quasi-two dimensional sheets (containing the $\beta$ and $\delta$ orbits) as well as a small inner 3D hole pocket centered at the Z-point $(\chi)$, shown in Fig.3D $[18,20,25]$. As a function of applied pressure, the cylindrical Fermi surfaces can become warped along the $k_{z}$ direction, either due to changes in the degree of interlayer hopping term and/or electronic correlations. These effects can trigger a Lifshitz transition and the disappearance of the neck orbits (like $\beta$ or $\alpha_{1}$ in Fig.3D), as found in $\mathrm{FeSe}_{1-x} \mathrm{~S}_{x}$ [19]. The multiband structure of $\mathrm{FeSe}_{0.89} \mathrm{~S}_{0.11}$ could give rise up to a maximum of seven frequencies, not all of which can be identified in experiment. The high pressure region is dominated by the largest orbit of the hole band, $\delta$, as shown in Fig. 2D (see also Ref. [38]). Weaker features associated to the largest orbit of the electron band, $\gamma$, can be also detected at some pressures and in the TDO signal, as shown in Fig. S7 in the SI.

With increasing pressure all frequencies increase in size, with the exception of the lowest two frequencies, as summarized in Fig. 3A. At the highest pressure, the Fermi surface area of the $\delta$ pocket has expanded almost by a factor two, as compared with its size at ambient pressure. This is much more than the expected growth of the Brillouin zone (less than $4 \%$ ), assuming a simple contraction of the in-plane unit cell $[14,39]$. On the other hand, the dominant low frequency, $\lambda_{1}$, (in Figs.2C, S4, S5, S6 in the SI), disappears together with nematicity close to $p_{c}$ (see $\lambda_{1}$ in Fig.3A). We attribute the loss of this frequency to a Lifshitz transition that can be triggered by changes in the interlayer coupling tuned by pressure. This observation is in agreement with the topological change of the Fermi surface using sulfur substitution, equivalent to positive pressure [19]. The presence of small pockets with Fermi energies of the order 3-5 meV in Fig. 3C, comparable to the superconducting gap (Fig. S6 in the SI), creates conditions for a BCS-BEC crossover [40].

Quasiparticle masses. We now turn to the evolution of the electronic correlations across the phase diagram. The evolution of the cyclotron effective mass, $m^{*}$, is extracted from the temperature dependence of the quantum oscillation amplitudes, analyzed quantitatively within the Lifshitz-Kosevich formalism (Fig. 2E) [29], and summarized in Fig. 3B. The quasiparticle masses of the $\delta$ orbit can be extracted across the entire pressure range, as shown in Fig. 2E, and its amplitude shows an unusual pressure dependence in Fig. $2 \mathrm{~F}$. The cyclotron mass of the largest frequency, $\delta$, (as well as $\chi$ and $\gamma$ in Fig. S7 in the SI), decreases significantly with increasing pressure from $5 m_{e}$ to $3 m_{e}$, signifying a strong reduction in electronic correlations away from the nematic phase transition. Thus, the quasiparticle masses gradually decrease rather
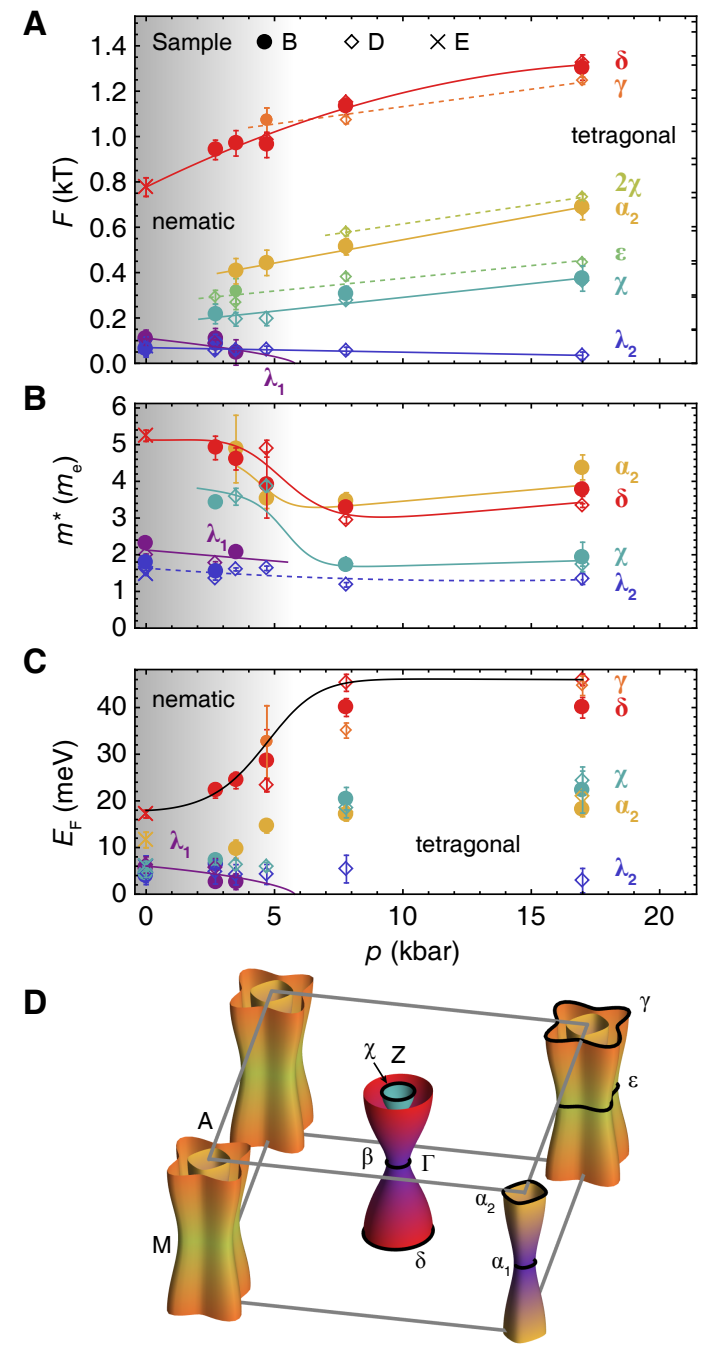

FIG. 3. Pressure tuning of Fermi surface and electronic correlations. (A) Quantum oscillation frequencies, and (B) the cyclotron masses as a function of pressure for samples B, D and E. The masses associated to $\chi$ and $\delta$ orbits show a clear decrease upon crossing the nematic phase transition. A topological Lifshitz transition occurs at $p_{c}$ and one small orbit in the $k_{z}=0$ plane disappears (either $\beta$ or $\alpha_{1}$ can be assigned to $\lambda_{1}$ ). A small frequency $\lambda_{2}$ is only detected in TDO and it seems weakly affected by pressure. (C) The Fermi energy $E_{F}$ assuming parabolic band dispersions, based on Fermi surface crosssections and quasiparticle masses (panels A and B). Lines are guides to the eye. The splitting of the $\gamma$ and $\delta$ frequencies is summarized in Fig. S7 in the SI. Shaded areas indicate the nematic phase (D) Sketch of the Fermi surface in the nematic phase and the different two-dimensional orbits, based on ARPES and quantum oscillation measurements [18-20]. Error bars correspond to a $1 \sigma$ confidence interval.

than showing a divergent behaviour across the nematic end point around $p_{c}$. In the high pressure regime, a slight increase in the effective masses of the $\delta$ and $\chi$ orbits is observed, but their values remain smaller than in the nematic phase, despite the fact that superconductivity has increased twice. Furthermore, the masses associated with $\gamma$ orbit, expected to contain $d_{x y}$ orbital character, is much heavier than $\delta$ orbit in FeSe 
$[17,25,41]$ but it cannot be clearly distinguished across the entire pressure phase diagram (as shown in Fig. S7 in the SI). However, the role of orbital-dependent electronic correlations cannot be fully assessed as the quasiparticle masses in quantum oscillations contain information about the cyclotron orbits that have averaged orbital character.

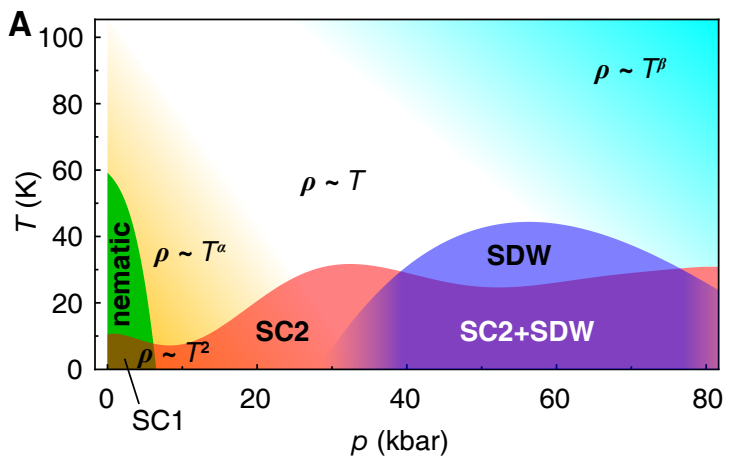

B

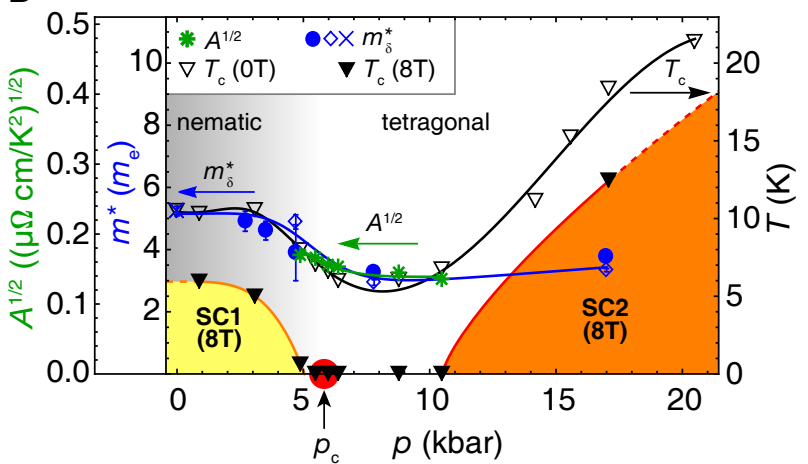

FIG. 4. Pressure-temperature phase diagrams. (A) Extended phase diagram of $\mathrm{FeSe}_{0.89} \mathrm{~S}_{0.11}$ based on our work and previous reports [22]. (B) Comparison of the band masses of the $\delta$ orbit with $T_{c}$ and the Fermi liquid coefficient, $A$. There is a qualitative link between the pressure dependence of electronic correlations and superconductivity across the nematic phase transition, which is lost towards the high pressure high- $T_{c}$ state. The temperature-pressure phase diagram in a static magnetic field of $8 \mathrm{~T}$ shows the separation of the two superconducting domes unmasking the nematic quantum phase transition at $p_{c}$. Symbols for the masses of the $\delta$ orbits are identical to Fig. 3. Solid lines are guides to the eye and dashed lines are extrapolations. Error bars correspond to a $1 \sigma$ confidence interval.

Discussion. The superconducting phase of $\mathrm{FeSe}_{0.89} \mathrm{~S}_{0.11}$ under pressure is composed of two distinct superconducting domes, separated by a Lifshitz transition. The first one at low pressure emerges from the nematic phase (SC1 in Fig.4B) and the second one at high pressure approaching the spin-density wave phase (SC2 in Figs.4A, B). The two domes can also be visualized in the pressure-temperature phase diagram in magnetic fields of $8 \mathrm{~T}$ in Fig.4B. Remarkably, the nematic quantum phase transition is located in the normal state between the two superconducting domes. Different types of superconducting paring inside and outside the nematic phase were suggested to occur in $\mathrm{FeSe}_{1-x} \mathrm{~S}_{x}$, based on STM, specific heat and thermal conductivity studies $[42,43]$.
The cyclotron effective masses, which measure the strength of electronic correlations, vary with pressure being in general larger inside the nematic phase compared with those in the tetragonal phase. Interestingly, we find a qualitative link between the cyclotron masses of the $\delta$ orbit, the $A$ coefficient from the low temperature resistivity data, and the value of $T_{c}$ in the nematic phase and in the low- $T_{c}$ tetragonal phase, as shown in Fig. 4B. This supports that the hole bands are closely involved in the pairing mechanism. This correlation between the band renormalisation and $T_{c}$ was also captured by quantum oscillations experiments and ARPES studies on $\mathrm{FeSe}_{1-x} \mathrm{~S}_{x}[19,20]$. Furthermore, NMR studies show that the strength of antiferromagnetic fluctuations also correlates with $T_{c}$ within the nematic phase, being suppressed together with the nematicity $[44,45]$. Here for higher pressures, the correlation between the hole-like band masses and $T_{c}$ is lost, suggesting changes in the pairing mechanism towards the high- $T_{c}$ phase. Furthermore, the observed Lifshitz transition of one of the pockets could also affect $T_{c}$, as it causes the density of states available for pairing to decrease. This decrease is likely to be weak and it is compensated by the contributions from other quasi-two dimensional Fermi surfaces that grow with pressure.

Upon approaching a critical region as a function of the tuning parameter, the quasiparticle masses $m^{*}[46]$ and the $A$ transport coefficient should diverge and the superconductivity should be enhanced $[32,37,46]$. Here, near the nematic end point at $p_{c}$, in the absence of magnetic order, we find a smoothly evolving $A$ coefficient and effective masses for the large, well-defined orbit $(\delta)$ in Fig. 4B. This suggests that the nematic fluctuations are finite and not critical at $p_{c}$. Additionally, the superconducting transition temperature is minimal in the vicinity of the nematic quantum phase transition at $p_{c}$. This implies that the suppression of superconductivity is connected with the lack of nematic critical fluctuations and/or spin fluctuations in this regime. Collapse of critical nematic fluctuations was observed in Raman measurements in FeSe under pressure, indicating they play a marginal role in stabilizing superconductivity [47]. This is in contrast to other ironbased superconductors where critical nematic and/or magnetic fluctuations enhance superconductivity approaching a spindensity wave state $[5,36]$.

The absence of divergent critical nematic fluctuations could suggest the presence of a coupling of the nematic order parameter with the shear mode of the lattice leading to quenched nematic criticality along certain directions $[27,48]$. This coupling could suppress superconductivity [48] and lead to a finite quasiparticle mass in the proximity of the putative nematic quantum critical point (compared with data in Fig. S7 in the SI). One test of this model is the predicted crossover from non-Fermi liquid behaviour at high temperatures towards a Fermi liquid at low temperatures, below $T_{F L}$. We can estimate that for our system $T_{F L} \sim 13 \mathrm{~K}$ which is in good agreement with the extracted values from our transport experiments ( $T_{F L} \sim 9-11 \mathrm{~K}$, as indicated by arrows in Fig.1D and detailed in Methods). Furthermore, the resistivity exponent $n$ 
close to $p_{c}$ in our study is not constant and it has a strong temperature dependence (Figs. 1B), as predicted for transport due the coupling with the lattice via acoustic phonons [49]. Similar transport behaviour and power laws reported here for $\mathrm{FeSe}_{0.89} \mathrm{~S}_{0.11}$ in the vicinity of the nematic end point were also found in $\mathrm{FeSe}_{1-x} \mathrm{~S}_{x}$ [50]. In any case, the transport behaviour near a nematic end point is very different from the linear $T$-behaviour in the proximity of a magnetic quantum critical point [36].

Additionally, the substitutional disorder of sulphur for selenium outside the Fe planes can lead to local strain that can potentially affect the nematic order parameter, acting as local fields and providing the realization of the random Ising model [51]. In this scenario, the induced local strains can set a limit of the correlation lengths and hence affect the nature of the nematic phase transition at low temperatures. While inherent substitutional disorder exist at ambient pressure (setting up the limit on the mean free path of $\sim 350 \AA$, as shown in Fig. S8 in the SI) the amount of disorder is a constant across the pressure-tuned nematic phase transition. Future theoretical models need to address how local strain (by STM as in Ref. [42]) combined with applied pressure affects critical behaviour in these types of systems. This approach could also provide insight into the significant suppression the amplitude of the quantum oscillation of the $\delta$ orbit inside the nematic phase beyond the normal damping terms due to disorder or the change in masses, as shown in Fig. 2F. Lastly, the nematic phase transition and the Lifshitz transition seem to coincide both with applied and chemical pressure [19]. The collapse of the critical nematic fluctuations and phonon anomalies were directly linked to the change in topology of the Fermi surface of FeSe under pressure from Raman studies [47].

The electronic signature of the nematic end point is substantially different from other critical points in iron-based superconductors, where both magnetic and nematic phases closely coexist and superconductivity is enhanced. The absence of divergent electronic correlations at the nematic phase transition invoke a coupling of the electronic system with the local environment. However, these effects are detrimental for supporting a high- $T_{c}$ phase, and consequently the pairing mechanism is weaken where spin fluctuations are absent.

* corresponding author:pascal.reiss@physics.ox.ac.uk

$\dagger$ corresponding author:amalia.coldea@physics.ox.ac.uk

[1] Sachdev, S. \& Keimer, B. Quantum criticality. Physics Today 64, 29 (2011).

[2] Schofield, A. J. Non-Fermi liquids. Contemporary Physics 40, 95-115 (1999).

[3] Pomeranchuk, I. I. On the Stability of a Fermi Liquid . Phys. Rev. Lett. 8, 361 (1958).

[4] Fernandes, R. M., Chubukov, A. V. \& Schmalian, J. What drives nematic order in iron-based superconductors? Nature Physics 10, 97104 (2014).

[5] Lederer, S., Schattner, Y., Berg, E. \& Kivelson, S. Superconductivity and non-Fermi liquid behavior near a nematic quan- tum critical point. Proc. Natl. Acad. Sci. USA 114, 4905 (2017).

[6] Sprau, P. O. et al. Discovery of Orbital-Selective Cooper Pairing in FeSe. Science 357, 75 (2016). URL https : / / arxiv . org/abs/1611.02134.

[7] Mizuguchi, Y., Tomioka, F., Tsuda, S., Yamaguchi, T. \& Takano, Y. Superconductivity at $27 \mathrm{~K}$ in tetragonal FeSe under high pressure. Appl. Phys. Lett. 93 (2008). 0807.4315.

[8] Medvedev, S. et al. Electronic and magnetic phase diagram of $\beta-\mathrm{Fe}_{1.01} \mathrm{Se}$ with superconductivity at $36.7 \mathrm{~K}$ under pressure. Nat. Mater. 8, 630 (2009). URL http: / / www. ncbi.nlm. nih.gov/pubmed/19525948.

[9] Terashima, T. et al. Pressure-Induced Antiferromagnetic Transition and Phase Diagram in FeSe. J. Phys. Soc. Japan 84, 063701 (2015). URL http: / / journals.jps.jp/doi/ abs/10.7566/JPSJ.84.063701.

[10] Terashima, T. et al. Fermi surface reconstruction in FeSe under high pressure. Phys. Rev. B 93, 094505 (2016). URL http://link.aps.org/doi/10.1103/ PhysRevB.93.094505.

[11] Wang, F., Kivelson, S. A. \& Lee, D.-H. Nematicity and quantum paramagnetism in FeSe. Nat. Phys. 11, 959 (2015).

[12] Wang, Q. et al. Strong interplay between stripe spin fluctuations, nematicity and superconductivity in FeSe. Nat. Mater. 15, 159 (2016). URL http://dx.doi. org/10.1038/nmat449210.1038/nmat4492http: //www.nature.com/nmat/journal/v15/n2/abs / nmat 4492 .html \{\#\} supplementary-information.

[13] Sun, J. P. et al. High- Tc Superconductivity in FeSe at High Pressure: Dominant Hole Carriers and Enhanced Spin Fluctuations. Phys. Rev. Lett. 118, 1 (2017).

[14] Kothapalli, K. et al. Strong cooperative coupling of pressureinduced magnetic order and nematicity in FeSe. Nat. Comm. 7, 12728 (2016). URL http://dx.doi.org/10.1038/ ncomms12728http://10.1038/ncomms12728.

[15] Imai, T., Ahilan, K., Ning, F. L., McQueen, T. M. \& Cava, R. J. Why does undoped FeSe become a high-Tc superconductor under pressure? Phys. Rev. Lett. 102, 1 (2009).

[16] Bendele, M. et al. Pressure Induced Static Magnetic Order in Superconducting $\mathrm{FeSe}_{1-x}$. Phys. Rev. Lett. 104, 087003 (2010). URL https://link.aps.org/doi/10.1103/ PhysRevLett.104.087003.

[17] Watson, M. D. et al. Emergence of the nematic electronic state in FeSe. Phys. Rev. B 91, 155106 (2015). URL http://link.aps.org/doi/10.1103/ PhysRevB.91.155106.

[18] Watson, M. D. et al. Suppression of orbital ordering by chemical pressure in $\mathrm{FeSe}_{1-x} \mathrm{~S}_{x}$. Phys. Rev. B 92, 121108 (2015). URL http://link.aps.org/doi/10.1103/ PhysRevB.92.121108.

[19] Coldea, A. I. et al. Evolution of the low-temperature Fermi surface of superconducting $\mathrm{FeSe}_{1-x} \mathrm{~S}_{x}$ across a nematic phase transition. npj Quantum Materials 4, 2 (2019). URL https://www nature.com/articles/ s41535-018-0141-0.

[20] Reiss, P. et al. Suppression of electronic correlations by chemical pressure from FeSe to FeS. Phys. Rev. B 96, 121103 (2017). URL https://link.aps.org/doi/10.1103/ PhysRevB.96.121103.

[21] Hosoi, S. et al. Nematic quantum critical point without magnetism in $\mathrm{FeSe}_{1-x} \mathrm{~S}_{x}$ superconductors. PNAS 113, 8139 (2016).

[22] Matsuura, K. et al. Maximizing $T_{c}$ by tuning nematicity and magnetism in $\mathrm{FeSe}_{1-x} \mathrm{~S}_{x}$ superconductors. Nat. Comm. 8, 1143 (2017). URL http: 
//arxiv.org/abs/1704.02057http://www. nature.com/articles/s41467-017-01277-x.

[23] Xiang, L. et al. Dome of magnetic order inside the nematic phase of sulfur-substituted FeSe under pressure. Phys. Rev. B 96, 024511 (2017). URL https : / / ink.aps.org/doi/ 10.1103/PhysRevB.96.024511.

[24] Yip, K. Y. et al. Weakening of the diamagnetic shielding in $\mathrm{FeSe}_{1-x} \mathrm{~S}_{x}$ at high pressures. Phys. Rev. B 96, 020502 (2017). URL http://arxiv.org/abs/1705.07639http: //link.aps.org/doi/10.1103/PhysRevB.96. 020502 .

[25] Coldea, A. I. \& Watson, M. D. The key ingredients of the electronic structure of FeSe. Annu. Rev. Cond. Matt. Phys. 9 (2018).

[26] Chubukov, A. V., Khodas, M. \& Fernandes, R. M. Magnetism, superconductivity, and spontaneous orbital order in iron-based superconductors: Which comes first and why? Phys. Rev. X 6, 041045 (2016). URL https:// link.aps.org/doi/ 10.1103/PhysRevX.6.041045.

[27] Paul, I. \& Garst, M. Lattice Effects on Nematic Quantum Criticality in Metals. Physical Review Letters 118, 1-5 (2017).

[28] Jaramillo, R., Feng, Y., Wang, J. \& Rosenbaum, T. F. Signatures of quantum criticality in pure cr at high pressure. Proceedings of the National Academy of Sciences 107, 13631-13635 (2010).

[29] Shoenberg, D. Magnetic Oscillations in Metals (Cambridge University Press, Cambridge, 1984).

[30] Rosch, A. Interplay of disorder and spin fluctuations in the resistivity near a quantum critical point. Phys. Rev. Lett. 82, 4280-4283 (1999). URL https: / / link. aps .org/doi/ 10.1103 /PhysRevLett. 82.4280.

[31] Maslov, D. L., Yudson, V. I. \& Chubukov, A. V. Resistivity of a Non-Galilean-Invariant Fermi Liquid near Pomeranchuk Quantum Criticality. Phys. Rev. Lett. 106, 106403 (2011). URL https: / / ink.aps.org/doi/10.1103/ PhysRevLett.106.106403.

[32] Löhneysen, H. v., Rosch, A., Vojta, M. \& Wölfle, P. Fermiliquid instabilities at magnetic quantum phase transitions. Rev. Mod. Phys. 79, 1015-1075 (2007). URL https: / / ink. aps.org/doi/10.1103/RevModPhys.79.1015.

[33] Oliver, G. T. Quantum multicriticality (2017). URL http: //etheses.bham.ac.uk/7179/.

[34] Wang, X. \& Berg, E. Scattering mechanisms and electrical transport near an ising nematic quantum critical point. Phys. Rev. B 99, 235136 (2019). URL https://link.aps. org/doi/10.1103/PhysRevB.99.235136.

[35] Licciardello, S. et al. Electrical resistivity across a nematic quantum critical point. Nature (2019). URL https: / / doi . org/10.1038/s41586-019-0923-y.

[36] Kasahara, S. et al. Evolution from non-Fermi- to Fermiliquid transport via isovalent doping in $\mathrm{BaFe}_{2}\left(\mathrm{As}_{1-x} \mathrm{P}_{x}\right)_{2}$ superconductors. Phys. Rev. B 81, 184519 (2010). URL https://link.aps.org/doi/10.1103/PhysRevB. 81.184519 .

[37] Analytis, J. G. et al. Transport near a quantum critical point in $\mathrm{BaFe}_{2}\left(\mathrm{As}_{1-x} \mathrm{P}_{x}\right)_{2}$. Nature Physics 10, 194 (2014).

[38] Watson, M. D. et al. Dichotomy between the Hole and Electron Behavior in Multiband Superconductor FeSe Probed by Ultrahigh Magnetic Fields. Phys. Rev. Lett. 115, 027006 (2015). URL http://link.aps.org/doi/10.1103/ PhysRevLett.115.027006.

[39] Tomita, T. et al. Correlation between $T_{c}$ and crystal structure in S-Doped FeSe superconductors under pressure: Studied by x-ray diffraction of $\mathrm{FeSe}_{0.8} \mathrm{~S}_{0.2}$ at Low Temperatures. Journal of the Physical Society of Japan 84, 1-8 (2015). URL https: //doi.org/10.7566/JPSJ.84.024713.
[40] Kasahara, S. et al. Field-induced superconducting phase of FeSe in the BCS-BEC cross-over. Proc. Natl. Acad. Sci. U. S. A. 111, 16309 (2014). URL http: //www.ncbi.nlm. nih.gov/pubmed/25378706.

[41] Terashima, T. et al. Anomalous Fermi surface in FeSe seen by Shubnikov-de Haas oscillation measurements. Phys. Rev. B 90, 144517 (2014). URL http: / / link .aps.org/doi/10. 1103 /PhysRevB.90.144517.

[42] Hanaguri, T. et al. Two distinct superconducting pairing states divided by the nematic end point in $\mathrm{FeSe}_{1-x} \mathrm{~S}_{x}$. Sci. Adv. 4, eaar6419 (2018).

[43] Sato, Y. et al. Abrupt change of the superconducting gap structure at the nematic critical point in $\mathrm{FeSe}_{1-x} \mathrm{~S}_{x}$. Proceedings of the National Academy of Sciences (2018). URL http: / / www.pnas.org/content/early/2018/01/ $22 / 1717331115$.

[44] Kuwayama, T. et al. Two different superconducting states and possible antiferromagnetic quantum critical points in S-doped FeSe under pressure. arXiv:1807.07118 (2018).

[45] Wiecki, P. et al. Persistent correlation between superconductivity and antiferromagnetic fluctuations near a nematic quantum critical point in $\mathrm{FeSe}_{1-x} \mathrm{~S}_{x}$. Phys. Rev. B 98, 020507 (2018). URL https://link.aps .org/doi/10.1103/ PhysRevB.98.020507.

[46] Shishido, H. et al. Evolution of the Fermi Surface of $\mathrm{BaFe}_{2}\left(\mathrm{As}_{1-x} \mathrm{P}_{x}\right)_{2}$ on Entering the Superconducting Dome. Phys. Rev. Lett. 104, 057008 (2010). URL https://link.aps.org/doi/10.1103/ PhysRevLett.104.057008.

[47] Massat, P. et al. Collapse of Critical Nematic Fluctuations in FeSe under Pressure. Phys. Rev. Lett. 121, 077001 (2018). URL https://link.aps .org/doi/10.1103/ PhysRevLett.121.077001.

[48] Labat, D. \& Paul, I. Pairing instability near a lattice-influenced nematic quantum critical point. Phys. Rev. B 96, 195146 (2017). URL https: / / link. aps .org/doi/10.1103/ PhysRevB.96.195146.

[49] de Carvalho, V. S. \& Fernandes, R. M. Resistivity near a nematic quantum critical point: impact of acoustic phonons. arXiv:1906.03205 (2019). URL https://arxiv.org/ abs/1906.03205.

[50] Bristow, M. et al. Anomalous high-magnetic field electronic state of the nematic superconductors $\mathrm{FeSe}_{1-x} \mathrm{~S}_{x}$ (2019). arXiv: 1904.02522.

[51] Carlson, E. W., Dahmen, K. A., Fradkin, E. \& Kivelson, S. A. Hysteresis and noise from electronic nematicity in hightemperature superconductors. Phys. Rev. Lett. 96, 097003 (2006). URL https://link.aps .org/doi/10.1103/ PhysRevLett.96.097003.

Acknowledgements We thank Penglin Cai for technical support provided with setting up the PPMS pressure cell and Andrey Chubukov, Erez Berg, Raphael Fernandes, Indranil Paul, Roser Valenti, Ilya Vekhter, Matthew Watson, Zachary Zajicek, Sid Parameswaran, Steve Simon for useful discussions and comments. This work was mainly supported by EPSRC (EP/I004475/1, EP/I017836/1). AAH acknowledges the financial support of the Oxford Quantum Materials Platform Grant (EP/M020517/1). A portion of this work was performed at the National High Magnetic Field Laboratory, which is supported by National Science Foundation Cooperative Agreement No. DMR-1157490 and the State of Florida. Part of 
this work was supported by HFML-RU/FOM and LNCMICNRS, members of the European Magnetic Field Laboratory (EMFL) and by EPSRC (UK) via its membership to the EMFL (grant no. EP/N01085X/1). Part of this work at the LNCMI was supported by Programme Investissements d'Avenir under the program ANR-11-IDEX-0002-02, reference ANR-10-LABX-0037-NEXT. AIC thanks the hospitality of KITP supported by the National Science Foundation under Grant No. NSF PHY- 1125915. We also acknowledge financial support of the John Fell Fund of the Oxford University. AIC acknowledges an EPSRC Career Acceleration Fellowship (EP/I004475/1).

Author contributions AIC proposed and supervised the project. PR, DG and AIC performed experiments in the hybrid magnet in Tallahassee. PR, WK, LD, MB, AIC performed experiments in pulsed fields in Toulouse. AAH grew the single crystals. AJS provided theoretical input. PR and AIC performed data analysis and wrote the paper with contributions and comments from all the authors.

Competing interests The authors declare no competing interest.

\section{Additional Information}

Supplementary information is available for this paper. Correspondence and requests for materials should be addressed to PR and AIC.

\section{Methods}

Sample characterization. Single crystals of $\mathrm{FeSe}_{0.89} \mathrm{~S}_{0.11}$ were grown by the $\mathrm{KCl} / \mathrm{AlCl}_{3}$ chemical vapour transport method, as reported previously $[52,53]$. More than 20 crystals were screened at ambient pressure and they showed sharp superconducting transition regions of $\sim 0.1 \mathrm{~K}$, and large residual resistivity ratios up to 17 between room temperature and the onset of superconductivity. Crystals from the same batch were previously used in quantum oscillations and ARPES studies $[17,19]$.

Magnetotransport measurements. High magnetic field measurements up to $45 \mathrm{~T}$ at ambient pressure and under hydrostatic pressure were performed using the hybrid magnet $\mathrm{dc}$ facility at the NHMFL in Tallahassee, FL, USA. Pressures up to $17 \mathrm{kbar}$ were generated using a $\mathrm{NiCoCr}$ piston cylinder cell, using Daphne Oil 7575 as pressure medium. The pressure inside the cell was determined by means of ruby fluorescence at low temperatures where quantum oscillations were observed. Different samples were measured simultaneously under pressure: sample B was used for transport measurements, and sample D-TDO was positioned inside a coil and the resonant frequency of an LC tank circuit driven by a tunnel diode was recorded (TDO). Transport sample E was measured at ambient pressure at the same time. Magnetotransport measurements were performed using the standard $a c$ technique. Pulsed-field measurements up to $80 \mathrm{~T}$ at ambient pressure were carried out in Toulouse, using the same samples (transport measurements on sample B, TDO measurements on sample D). Magnetotransport and Hall effect measurements under pressure using a 5-contact configuration were carried out on sample A in low fields up to 16 T in an Oxford Quantum Design PPMS and an ElectroLab High Pressure Cell, using Daphne Oil 7373 which ensures hydrostatic conditions up to about $23 \mathrm{kbar}$. The pressure inside this cell was determined via the superconducting transition temperature of Sn after cancelling the remanent field in the magnet. The magnetic field was applied along the crystallographic $c$ axis for all samples. A maximum current of up to $2 \mathrm{~mA}$ flowing in the conducting tetragonal $a b$ plane was used.

Resistivity power law. The temperature and pressure dependence of $n$ is defined as $\left.n=\partial \log \left(\rho-\rho_{0}\right) / \partial \log T\right)$ with $\rho_{0}$ extrapolated from low temperatures. To extrapolate the zero-field resistivity at finite temperature, $\rho_{0}(T)$, in the absence of superconductivity (Fig. S2 in the SI), we perform a two-band model fit to the symmetrized magnetotransport data [54].

Estimate for $T_{F L}$. For a system with a strong nematoelastic coupling, this interaction leads to an enhanced nematic transition temperature $T_{s}>T_{0}$, where $T_{0}$ is the bare transition temperature in its absence $[27,48]$. Furthermore, below a crossover temperature $T_{F L}$, the coupling quenches the critical modes to small regions in $k$-space which will lead to Fermi liquid behaviour. In order to estimate the scale of $T_{F L}$, it is expected that the nematic quantum critical point is shifted to a larger tuning parameter $r_{0}>0$ caused by the nematoelastic coupling [27]. This shift is linearly related to $T_{s}$ and $T_{0}$ as $r_{0} \approx k_{B}\left(T_{s}-T_{0}\right) / E_{F}$, where $E_{F}$ is the Fermi energy and $k_{B}$ is the Boltzmann constant. The value of $T_{0}$ can be estimated from electronic Raman scattering [27] or elastoresistivity measurements [21]. For FeSe, $T_{0} \approx 30 \mathrm{~K}$ from electronic Raman scattering [55] and $T_{0} \approx 34 \mathrm{~K}$ from elastoresistivity measurements [21]. For $\mathrm{FeSe}_{0.89} \mathrm{~S}_{0.11}$, we use the ambient pressure values $T_{0}=15 \mathrm{~K}$ and $T_{s}=60 \mathrm{~K}$ (see also Fig. S1 in the SI) and a Fermi energy of $E_{F} \approx 50 \mathrm{meV}$ [18]. We thus obtain $r_{0} \approx 0.077 \ll 1$, comparable to estimates for other iron-based superconductors [27]. From this, we can determine $T_{F L}$ using $T_{F L} \approx r_{0}^{3 / 2} E_{F} / k_{B}$ [27]. We find that $T_{F L} \approx 13 \mathrm{~K}$ for $\mathrm{FeSe}_{0.89} \mathrm{~S}_{0.11}$. This value is well within the experimentally accessible temperature range and it is consistent with the crossover region of the resistivity exponent $n$ at $T_{F L}$, shown in Fig. 1B (also Fig. S1D in the SI).

Quantum oscillations. To quantify the complex oscillatory spectra of quantum oscillations, we use both a fast Fourier transform (after removing a smooth and monotonic polynomial background from the raw data) or directly fitting the Lifshitz-Kosevich formalism to the raw data in field (with a low-pass filter applied), as shown in Figs. 2C, S4 and S6 in the SI. The frequencies of the quantum oscillations are related to the extremal areas on the Fermi surface normal to the applied magnetic field, $A_{k}$, via the Onsager relation, $F=A_{k} \hbar /(2 \pi e)$ [29].

Data availability The experimental data in our manuscript will be made available available through 
ORA depository at the University of Oxford at https://doi.org/10.5287/bodleian:2REyEPKZX. Other information and requests for this study are available from the corresponding authors upon reasonable request.

* corresponding author:pascal.reiss@ physics.ox.ac.uk

$\dagger$ corresponding author:amalia.coldea@physics.ox.ac.uk

[52] Böhmer, A. E. et al. Lack of coupling between superconductivity and orthorhombic distortion in stoichiomet- ric single-crystalline FeSe. Phys. Rev. B 87, 180505 (2013). URL http://link.aps.org/doi/10.1103/ PhysRevB. 87.180505.

[53] Böhmer, A. E., Taufour, V., Straszheim, W. E., Wolf, T. \& Canfield, P. C. Variation of transition temperatures and residual resistivity ratio in vapor-grown FeSe. Phys. Rev. B 94, 024526 (2016). URL https://link.aps .org/doi/10.1103/ PhysRevB. 94.024526.

[54] Proust, C., Vignolle, B., Levallois, J., Adachi, S. \& Hussey, N. E. Fermi liquid behavior of the in-plane resistivity in the pseudogap state of $\mathrm{YBa}_{2} \mathrm{Cu}_{4} \mathrm{O}_{8}$. Proceedings of the National Academy of Sciences 113, 13654-13659 (2016).

[55] Massat, P. et al. Proc. Natl. Acad. Sci. 113, 9177 (2016). 